\title{
Colorectal carcinoma and chronic inflammatory demyelinating polyneuropathy: is there a possible paraneoplastic association?
}

\author{
Adnan Malik ${ }^{1}$, Faisal Inayat ${ }^{2}$, Muhammad Hassan Naeem Goraya ${ }^{3}$, Gul Nawaz ${ }^{4}$, Ahmad Mehran $^{2}$, Atif Aziz ${ }^{5}$, Saad Saleem ${ }^{6}$ \\ ${ }^{1}$ Loyola University Medical Center, Maywood, IL, USA; ${ }^{2}$ Allama Iqbal Medical College, Lahore; ${ }^{3}$ Services Institute of Medical Sciences, Lahore, Pakistan; \\ ${ }^{4}$ Marshfield Clinic, Marshfield, WI, USA; ${ }^{5}$ Islamic International Medical College, Rawalpindi, Pakistan; ${ }^{6}$ Sunrise Hospital and Medical Center, Las Vegas, NV, \\ USA
}

A plethora of paraneoplastic syndromes have been reported as remote effects of colorectal carcinoma (CRC). However, there is a dearth of data pertaining to the association of this cancer with demyelinating neuropathies. Herein, we describe the case of a young woman diagnosed with chronic inflammatory demyelinating polyneuropathy (CIDP). Treatment with intravenous immunoglobulins and prednisone did not improve her condition, and her neurological symptoms worsened. Subsequently, she was readmitted with exertional dyspnea, lightheadedness, malaise, and black stools. Colonoscopy revealed a necrotic mass in the ascending colon, which directly invaded the second part of the duodenum. Pathologic results confirmed the diagnosis of locally advanced CRC. Upon surgical resection of the cancer, her CIDP showed dramatic resolution without any additional therapy. Patients with CRC may develop CIDP as a type of paraneoplastic syndrome. Clinicians should remain cognizant of this potential association, as it is of paramount importance for the necessary holistic clinical management.

Keywords: Chronic inflammatory demyelinating polyneuropathy; Colorectal neoplasms; Paraneoplastic syndromes

\section{INTRODUCTION}

According to the World Health Organization GLOBOCAN database, 1.8 million new cases of colorectal carcinoma (CRC) and 861,000 deaths were recorded in 2018. ${ }^{1}$ The knowledge about different types of paraneoplastic syndromes associated with this globally ubiquitous cancer is imperative. In addition to other manifestations, a wide range of peripheral neuropathies

\section{Received: February 6, 2021 Revised: March 29, 2021}

Accepted: April 5, 2021

Correspondence: Faisal Inayat

Allama Iqbal Medical College, Allama Shabbir Ahmad Usmani Road, Faisal Town, Lahore 54550, Pakistan

E-mail: faisalinayat@hotmail.com

(a) This is an Open Access article distributed under the terms of the Creative Commons Attribution Non-Commercial License (http://creativecommons.org/licenses/by-nc/4.0/) which permits unrestricted non-commercial use, distribution, and reproduction in any medium, provided the original work is properly cited. have also been reported as remote effects of CRC. However, paraneoplastic demyelinating neuropathies are rarely observed. Chronic inflammatory demyelinating polyneuropathy (CIDP) is an acquired immune-mediated inflammatory disease characterized by inflammation and destruction of the myelin sheath in the peripheral nervous system, with an estimated prevalence of up to 10.3 per 100,000 individuals. ${ }^{2,3}$ While CIDP has been associated with various malignancies, a paraneoplastic correlation between CRC and CIDP remains rarely recognized. ${ }^{4}$ Consequently, the pathophysiological link between these illnesses and the clinical outcomes of their co-occurrence has not been well-studied. In the current study, we describe the case of a patient with chronic CIDP who was subsequently diagnosed with infiltrating CRC. This article illustrates that updated knowledge of this association will help clinicians promptly investigate patients with CIDP for an underlying CRC. Furthermore, it will enable existing CRC patients to undergo stringent clinical sur- 
veillance for potential CIDP symptoms, facilitating early treatment for neuropathy.

\section{CASE REPORT}

A 32-year-old woman was diagnosed with CIDP on a standard set of investigations on May 25, 2018. She was immediately initiated on monthly intravenous immunoglobulin (IVIG) $(1 \mathrm{~g} / \mathrm{kg})$ therapy. She received a total of 10 cycles, with only a transient improvement in strength after each course. Subsequently, her condition worsened, and she gradually became unresponsive to IVIG treatment. She was then administered $10 \mathrm{mg}$ prednisone, achieving only brief symptomatic relief. Two months before her current presentation, she was diagnosed with new-onset mild anemia (hemoglobin, $11 \mathrm{~g} / \mathrm{dL}$ ). Her primary care physician performed an assessment and started iron replacement therapy, considering it to be related to her menstrual bleeding. The patient was admitted to our hospital for the evaluation of dyspnea on exertion, lightheadedness, malaise, and black stools developed over the past week. She reported episodic epigastric pain, occasional nausea, bloating, and constipation. At presentation, the patient was hemodynamically stable. Physical examination revealed conjunctival pallor with mild generalized abdominal discomfort, while a clinical examination detected progressive sensorimotor neuropathy affecting all four limbs with diffuse areflexia.

Blood tests conducted upon admission revealed the following levels: hemoglobin $5.4 \mathrm{~g} / \mathrm{dL}$, hematocrit $19.8 \%$, red cell count $3.43 \times 10^{6} / \mu \mathrm{L}$, mean corpuscular volume $56.8 \mathrm{fL}$, mean corpuscular hemoglobin $16.3 \mathrm{pg} / \mathrm{cell}$, and mean corpuscular hemoglobin concentration $25.7 \mathrm{~g} / \mathrm{dL}$, indicating severe hypochromic microcytic anemia. Her white cell and platelet counts, renal and liver function tests, thyroid profile, vitamin B12, and serum electrolytes were within normal limits. The tests for celiac disease and Helicobacter pylori infection were negative. Anti-myelin-associated glycoprotein (anti-MAG) antibodies tested positive in the immunofluorescence assay. Esophagogastroduodenoscopy revealed an obstructing mass in the second part of the duodenum (Fig. 1A). Histopathological analysis of the biopsy specimen confirmed a moderately differentiated adenocarcinoma. Her colonoscopy also showed a large necrotic mass in the ascending colon, which was endoscopically consistent with malignancy (Fig. 1B). Biopsy specimens were sent for histopathology, which confirmed the presence of infiltrating colorectal adenocarcinoma. Abdominal computed tomography was performed to exclude two different cancers. It revealed that the ascending colon mass directly invaded the duodenum (Fig. 2). No abdominal or thoracic distant tumor spread was noted on computed tomography scans.

Subsequently, the patient underwent an exploratory laparotomy. It confirmed that the ascending colon cancer involving the hepatic flexure, was invading the second portion of the duodenum. A right hemicolectomy plus pancreaticoduodenectomy was performed, with no intra-operative complications. The postprocedural hospital course was unremarkable, and the patient showed good clinical recovery. She was discharged
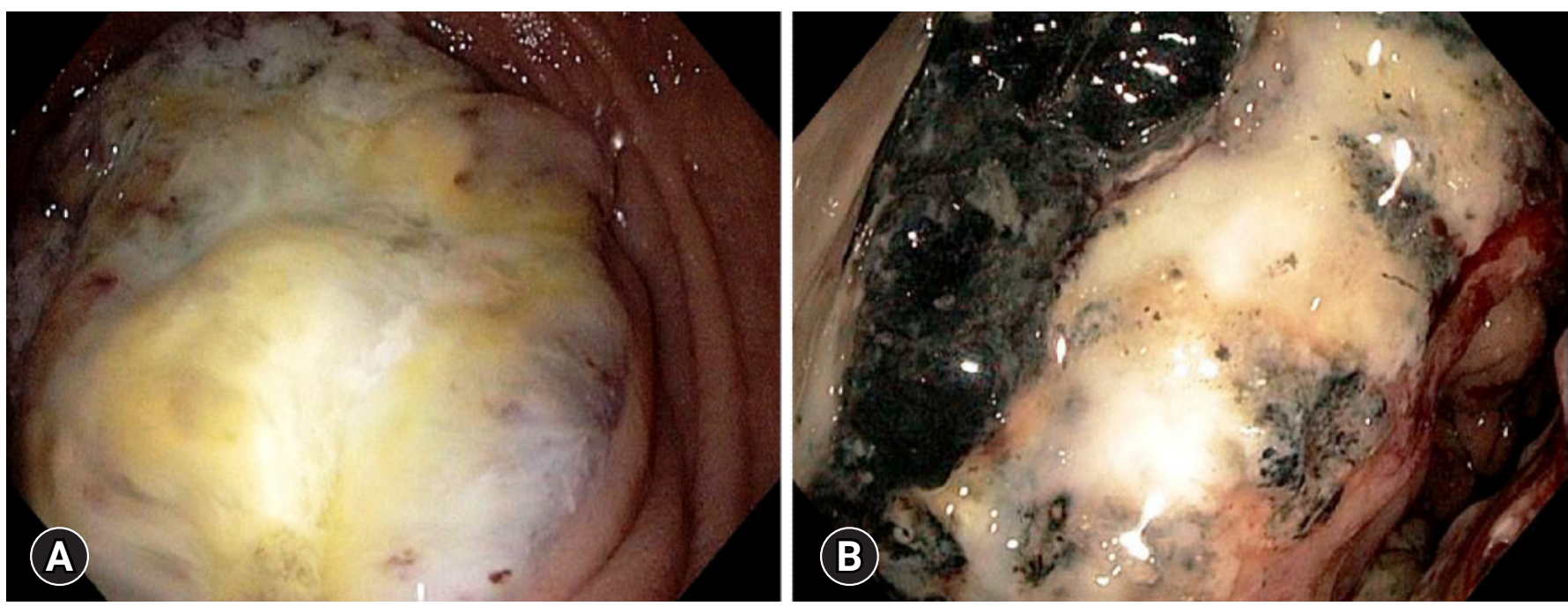

Fig. 1. Endoscopic evaluation. (A) Upper endoscopy showing a mass in the second part of the duodenum. (B) Colonoscopy showing a large, necrotic mass in the ascending colon. 

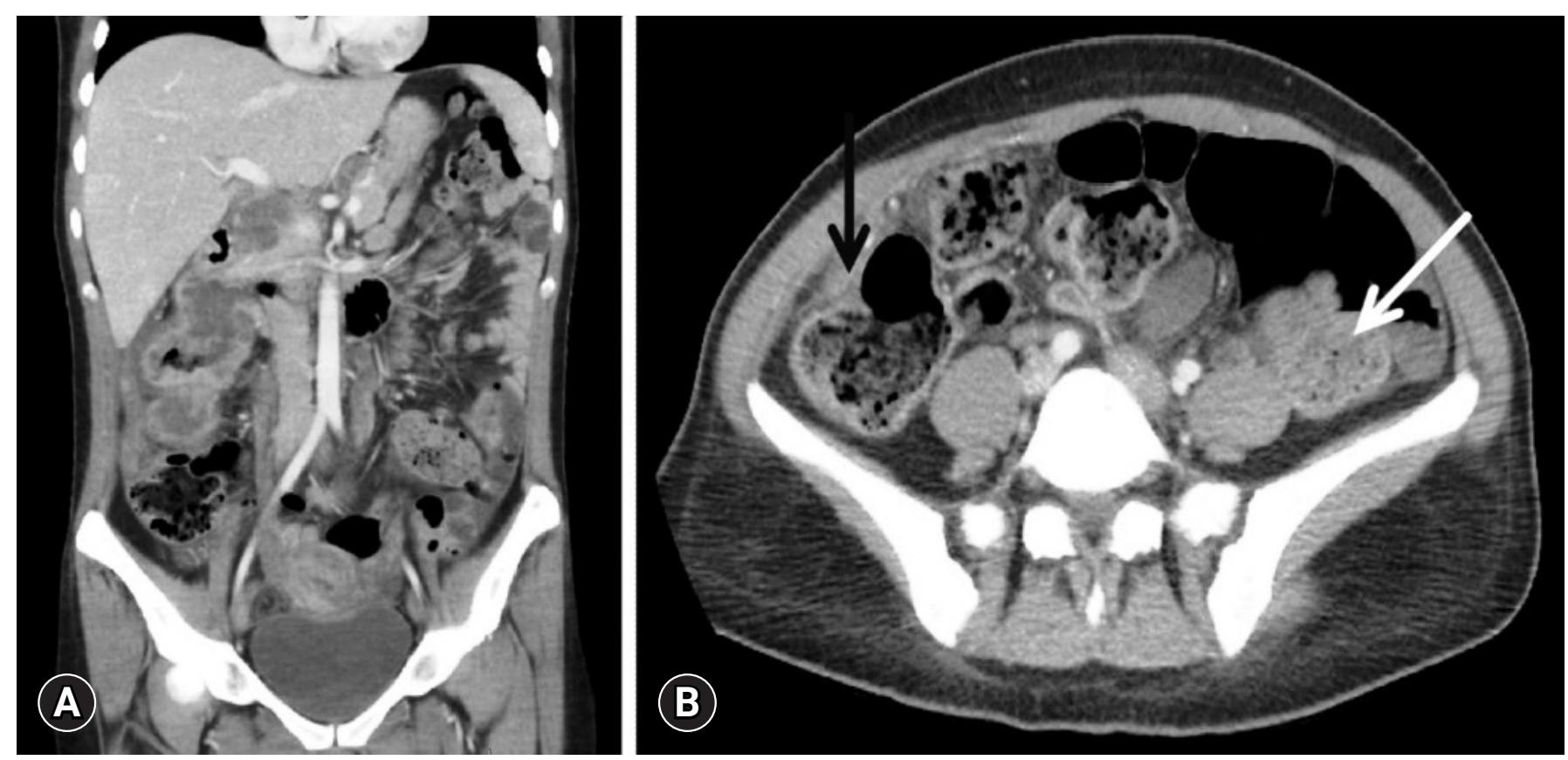

Fig. 2. Abdominopelvic contrast-enhanced computed tomography findings. (A) Coronal reformatted section showing a large circumferential heterogeneously enhancing necrotic mass involving the ascending colon up to the hepatic flexure, causing significant luminal narrowing. The superior component of the mass is infiltrating into the descending portion of the duodenum. (B) Axial section showing abnormal wall thickening of the large bowel, more pronounced on the lateral side of the ascending colon, with significant fat stranding (black arrow). Dilated small bowel loop along with fecal loading due to the obstruction caused by the tumor mass (white arrow).

from the hospital on postoperative day 12 in a stable condition. Pathologic assessment demonstrated a right colonic moderately differentiated adenocarcinoma with duodenal and pancreatic head invasion and tumor-free surgical resection margins. Lymphatic involvement was not noted.

Immunohistochemical examination confirmed the colonic origin of the cancer. According to pathological reports, the stage of CRC was IIC (T4bN0M0), with high-risk features. As per the National Comprehensive Cancer Network (NCCN) guidelines, adjuvant chemotherapy with leucovorin and 5-fluorouracil was initiated. In terms of CIDP-related abnormalities, the patient showed clear improvement after bowel resection. At the 10-month follow-up, nerve conduction studies revealed resolution of all demyelinating features, with marked overall strength improvement. Anti-MAG antibodies disappeared in the subsequent biochemical testing.

\section{DISCUSSION}

A wide range of paraneoplastic syndromes has previously been described in patients with CRC (Table 1). However, to our knowledge, the association with CIDP remains extremely rare. ${ }^{5}$ We conducted a systematic search of MEDLINE, Cochrane, Embase, and Scopus databases from inception until their latest publications. Search terminologies such as "colorectal adenocarcinoma", "colon cancer" and "paraneoplastic syndrome" were combined using the Boolean operators "AND" and "OR" with the terms "neuropathy" and "chronic inflammatory demyelinating polyneuropathy". The search methodology for data synthesis and the results are shown in Fig. 3. After exclusion of duplicate and redundant search results, we found only three previously reported cases of CIDP associated with CRC (Table 2) ${ }^{6-8} \mathrm{~A}$ comprehensive review of these cases revealed that two patients were male and one was female, ranging in age from 53-78 years. One patient developed CIDP concurrently with CRC detection, while two were affected from the neuropathy months to years before the cancer diagnosis.

Published medical literature remains scarce on the association between CRC and CIDP being a coincidence or part of a paraneoplastic syndrome. Prior research suggests that carcinomas may show a propensity to produce certain antigens on cancer cells that are shared with Schwann cells. ${ }^{9}$ Therefore, such 
Table 1. Paraneoplastic syndromes associated with colorectal cancer

\begin{tabular}{|c|c|c|c|c|c|}
\hline Dermatological & Rheumatic & Renal & Hematological & $\begin{array}{c}\text { Central } \\
\text { nervous system }\end{array}$ & $\begin{array}{c}\text { Peripheral } \\
\text { nervous system }\end{array}$ \\
\hline $\begin{array}{l}\text { Acquired hypertrichosis } \\
\text { lanuginose }\end{array}$ & $\begin{array}{l}\text { Carcinomatous } \\
\text { polyarthritis }\end{array}$ & $\begin{array}{l}\text { Membranous } \\
\text { glomerulopathy }\end{array}$ & Venous thromboembolism & $\begin{array}{l}\text { Optic neuropathy } \\
\text { and retinopathy }\end{array}$ & $\begin{array}{l}\text { Lambert-Eaton } \\
\text { myasthenic } \\
\text { syndrome }\end{array}$ \\
\hline Paraneoplastic acrokeratosis & $\begin{array}{l}\text { Palmar fasciitis } \\
\text { and arthritis }\end{array}$ & MPGN & Migratory thrombophlebitis & Limbic encephalitis & $\begin{array}{l}\text { Stiff person } \\
\text { syndrome }\end{array}$ \\
\hline Paraneoplastic pemphigus & $\begin{array}{l}\text { Multicentric } \\
\text { reticulohistiocytosis }\end{array}$ & SIADH & Paraneoplastic eosinophilia & PCD & $\begin{array}{l}\text { Sensory-motor } \\
\text { polyneuropathy }\end{array}$ \\
\hline Paraneoplastic dermatomyositis & RS3PE & $\begin{array}{l}\text { Hypercalcemia } \\
\text { of malignancy }\end{array}$ & Hemolytic anemia & $\begin{array}{l}\text { Necrotizing } \\
\text { myelopathy }\end{array}$ & Polymyositis \\
\hline Erythema gyratum repens & $\begin{array}{l}\text { Polymyalgia } \\
\text { rheumatic }\end{array}$ & $\begin{array}{l}\text { Cushing's } \\
\text { syndrome }\end{array}$ & & & \\
\hline Erythroderma & $\begin{array}{l}\text { Raynaud's } \\
\text { phenomenon }\end{array}$ & & & & \\
\hline \multicolumn{6}{|l|}{$\begin{array}{l}\text { Cutaneous leukocytoclastic } \\
\text { vasculitis }\end{array}$} \\
\hline Sweet's syndrome & & & & & \\
\hline
\end{tabular}

MPGN, membranoproliferative glomerulonephritis; SIADH, syndrome of inappropriate antidiuretic hormone secretion; PCD, paraneoplastic cerebellar degeneration; RS3PE, remitting seronegative symmetrical synovitis with pitting edema.

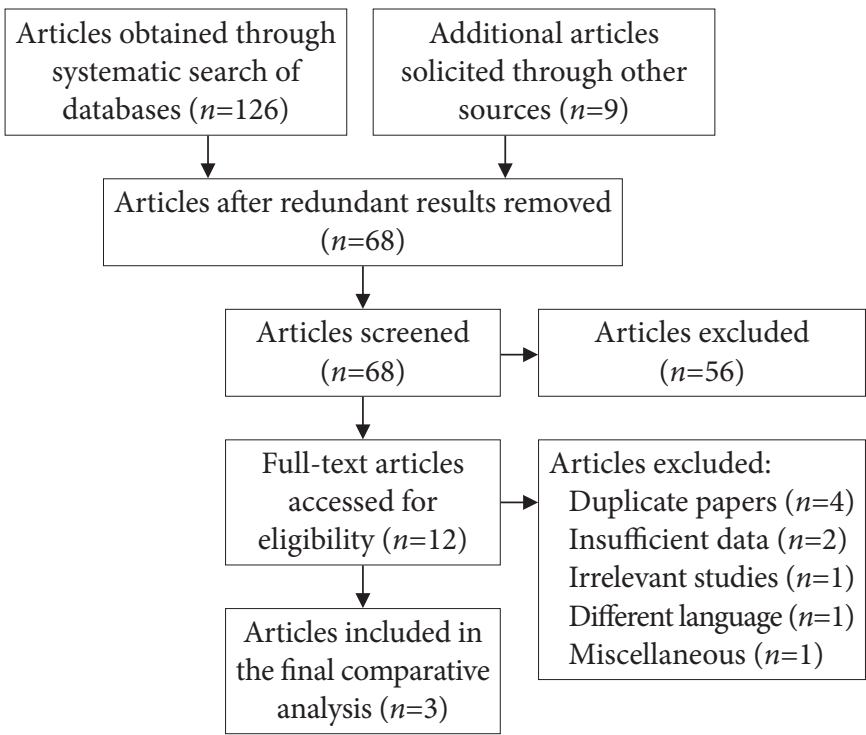

Fig. 3. Flow diagram representing the search methodology for data synthesis regarding the association of colorectal carcinoma with chronic inflammatory demyelinating polyneuropathy.

cancers may trigger the production of onconeural antibodies that may also cross-react with the aforementioned nerve cells, ultimately causing CIDP. $^{9-11}$ Although immunotherapy can trigger CIDP in certain malignancies, all three cases of CRC-CIDP were reported either at or prior to the cancer diagnosis. ${ }^{6-8,12}$ Ayyappan et al. ${ }^{7}$ reported a case of distally acquired demyelin- ating symmetric (DADS) neuropathy. They argued that CRC triggered an immune response, leading to the production of anti-MAG antibodies. When the antigenic burden in the form of CRC was surgically removed, immune stimulation and production of antibodies were notably reduced. This led to the clinical and electrophysiological recovery of CIDP with cancer treatment. ${ }^{7}$ Galassi and Luppi $^{8}$ reported a similar patient with acroparesthesia who failed to respond to IVIG therapy and was subsequently found to have CRC. However, they were skeptical about this particular pathological association.

In our patient, the etiological mechanism behind CIDP is likely to be a paraneoplastic syndrome due to the close temporal relationship between cancer resection and resolution of clinical symptomology, marked improvement of overall strength, normalization of electrophysiological patterns, and the disappearance of anti-MAG antibodies. Notably, demyelinating neuropathy does not usually improve without immunotherapy. However, this patient did not receive any additional therapy for CIDP, and she achieved dramatic clinical and electrophysiological recovery. Furthermore, anti-MAG antibodies may persist for a longer period of time in these patients. However, in our case, it was not detected soon after tumor resection. According to the definition of a paraneoplastic syndrome, CIDP was indeed a heterogeneous disorder that preceded the CRC diagnosis and was not physically related to the tumor or its metastasis. ${ }^{13}$ It was independent of local tumor effects, metabolic and nutritional 


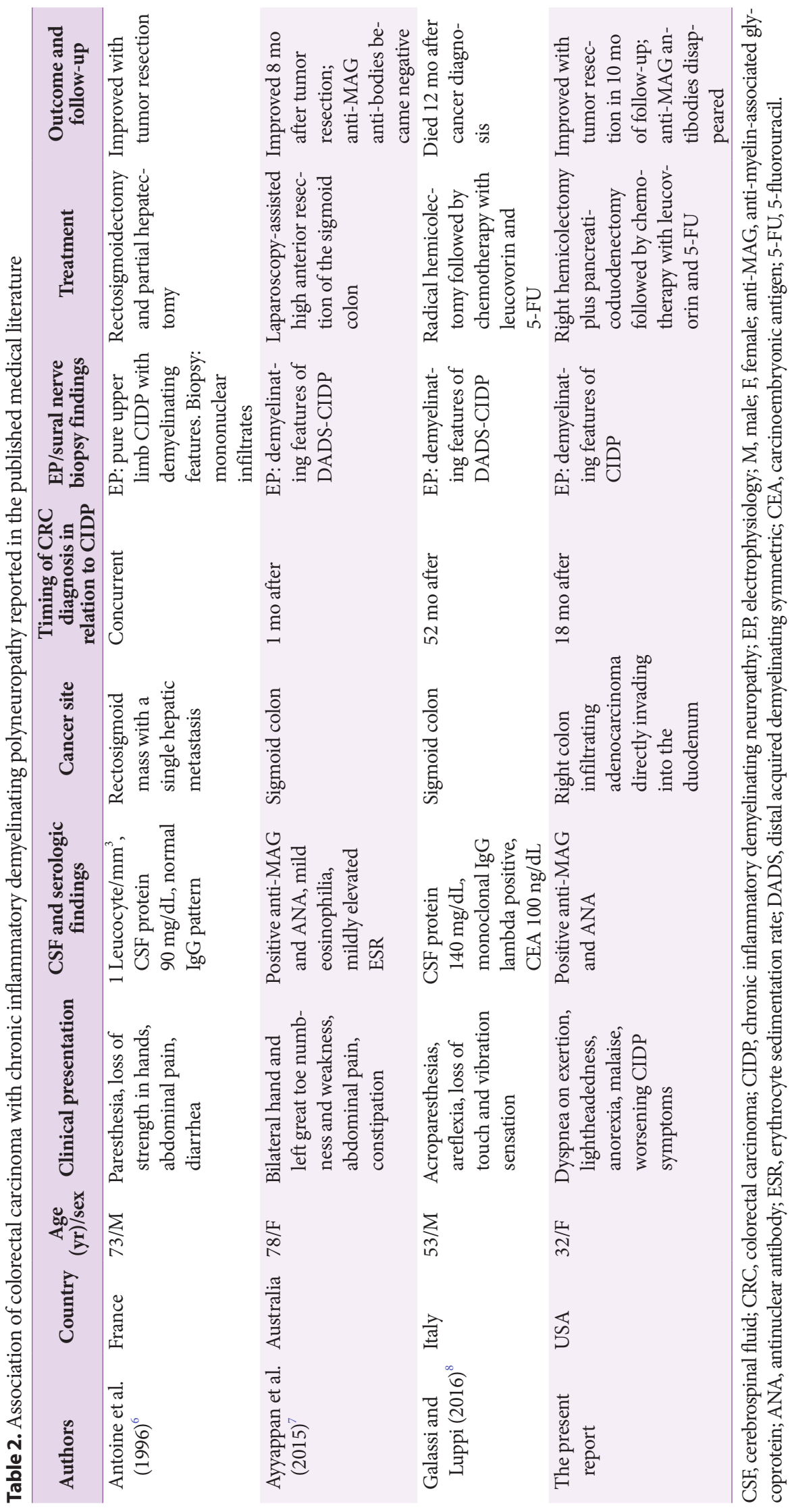


deficits, infection, coagulopathy, and cancer chemotherapy. ${ }^{14}$ These observations suggest the potential existence of a causal paraneoplastic mechanism between CRC and CIDP.

With regard to the diagnosis of CRC, a multitude of stoolbased tests and visual examination of the colon and rectum are recommended for screening and detection. CIDP is suspected based on the initial history and clinical examination. After exclusion of other probable etiologies, the diagnostic confirmation is primarily based on the evidence of peripheral nerve demyelination on electrodiagnostic results or rarely by nerve biopsy. ${ }^{15}$ The European Federation of Neurological Societies/ Peripheral Nerve Society (EFNS/PNS) guideline classifies CIDP as typical (classic) or atypical. ${ }^{16}$ Clinical inclusion criteria for typical CIDP requires either chronically progressive, stepwise, or recurrent symmetric proximal and distal weakness or sensory dysfunction of all extremities, developing over at least 2 months or absent/reduced tendon reflexes in all extremities. ${ }^{16}$ In patients with atypical CIDP, diagnosis requires one of the following neuropathic features: predominantly distal, asymmetric, focal, pure motor, or pure sensory. ${ }^{16}$ Based on EFNS/PNS criteria, CIDP can also be classified into three categories as definite, probable, and possible. ${ }^{16}$ The sensitivity of the EFNS/PNS criteria is $81 \%$ to $99 \%$, and the specificity is $61 \%$ to $97 \%$. $^{17}$

In patients with CRC, CIDP may resolve after complete remission of cancer. Therefore, priority should be given to the treatment of the malignancy itself and should not be delayed unless necessary. If cancer treatment does not resolve neurological complaints, immunotherapy may be initiated. Therapeutic modalities such as corticosteroids, plasma exchange, and/or IVIG therapy are generally successful in treating cancer-related CIDP. ${ }^{18}$ In the CIDP-CRC cases described in the literature, the surgical resection of CRC with or without adjuvant chemotherapy frequently resulted in the resolution of CIDP. ${ }^{6-8}$ In the current patient, the symptoms of CIDP showed remarkable improvement with surgical intervention alone. However, due to stage IIC (T4bN0M0) CRC with high-risk characteristics, adjuvant chemotherapy was administered in accordance with the NCCN guidelines. ${ }^{19}$ As the underlying etiology governs the eventual management in patients with CRC-associated CIDP, early recognition of a paraneoplastic syndrome also carries paramount therapeutic importance. It spares these patients from going through multiple evaluations and hospital visits, saving valuable hospital resources. ${ }^{20}$ Furthermore, if the diagnosis of CIDP precedes CRC detection, it may facilitate early detection and prompt initiation of appropriate treatment, which in turn may help to lower the potential morbidity and mortality associated with a delayed cancer diagnosis.

In conclusion, this case report describes the unusual co-existence of CRC and CIDP in a patient whose initial clinical presentation was dominated by neurological symptoms related to CIDP. It offers a clinical basis for previous reports suggesting a possible paraneoplastic association between these disorders and provides evidence that tumor resection may be a potential treatment strategy for medically refractory CIDP in such patients.

\section{Conflicts of Interest}

The authors have no potential conflicts of interest.

\section{Funding}

None.

\section{Author Contributions}

Conceptualization: all authors; Data curation: AM, FI, MHNG; Formal analysis: AM, FI; Investigation: FI, AM, AA, SS; Methodology: all authors; Project administration: AM, FI; Visualization: all authors; Writing-original draft: AM, FI, MHNG; Writing-review \& editing: all authors.

\section{ORCID}

Adnan Malik

Faisal Inayat

https://orcid.org/0000-0002-4276-205X

Muhammad Hassan Naeem Goraya

https://orcid.org/0000-0003-4826-6580

Gul Nawaz https://orcid.org/0000-0002-4978-4761

Ahmad Mehran

Atif Aziz https://orcid.org/0000-0002-9064-4242

Saad Saleem https://orcid.org/0000-0001-5276-1985 https://orcid.org/0000-0002-1327-492X

\section{REFERENCES}

1. Bray F, Ferlay J, Soerjomataram I, et al. Global cancer statistics 2018: GLOBOCAN estimates of incidence and mortality worldwide for 36 cancers in 185 countries. CA Cancer J Clin 2018;68:394-424.

2. Dyck PJ, Lais AC, Ohta M, et al. Chronic inflammatory polyradiculoneuropathy. Mayo Clin Proc 1975;50:621-637.

3. Broers MC, Bunschoten C, Nieboer D, et al. Incidence and prevalence of chronic inflammatory demyelinating polyradiculoneuropathy: a systematic review and meta-analysis. Neuroepidemiology 2019;52:161-172. 
4. Malik A, Berry R, Fung BM, et al. Association between chronic inflammatory demyelinating polyneuropathy and gastrointestinal malignancies. Clin J Gastroenterol 2021;14:1-13.

5. Rajabally YA, Attarian S. Chronic inflammatory demyelinating polyneuropathy and malignancy: a systematic review. Muscle Nerve 2018;57:875-883.

6. Antoine JC, Mosnier JF, Lapras J, et al. Chronic inflammatory demyelinating polyneuropathy associated with carcinoma. J Neurol Neurosurg Psychiatry 1996;60:188-190.

7. Ayyappan S, Day T, Kiers L. Distal acquired demyelinating symmetric (DADS) neuropathy associated with colorectal adenocarcinoma. Muscle Nerve 2015;51:928-931.

8. Galassi G, Luppi G. Distal acquired demyelinating symmetric neuropathy associated with colorectal adenocarcinoma: should it be termed paraneoplastic? Muscle Nerve 2016;53:658-659.

9. Bell CE, Seetharam S. Identification of the Schwann cell as a peripheral nervous system cell possessing a differentiation antigen expressed by a human lung tumor. J Immunol 1977;118:826-831.

10. Kudo M, Noguchi T. Immunoreactive myelin basic protein in tumor cells associated with carcinomatous neuropathy. Am J Clin Pathol 1985;84:741-743.

11. Altermatt HJ, Rodriguez M, Scheithauer BW, et al. Paraneoplastic anti-Purkinje and type I anti-neuronal nuclear autoantibodies bind selectively to central, peripheral, and autonomic nervous system cells. Lab Invest 1991;65:412-420.

12. Fuller GN, Spies JM, Pollard JD, et al. Demyelinating neuropathies triggered by melanoma immunotherapy. Neurology 1994;44:2404-
2405.

13. Henry K. Paraneoplastic syndromes: definitions, classification, pathophysiology and principles of treatment. Semin Diagn Pathol 2019;36:204-210.

14. Jameson JL, Longo DL. Paraneoplastic syndromes: endocrinologic/ hematologic. In: Jameson JL, Fauci AS, Kasper DL, Hauser SL, Longo DL, Loscalzo J, editors. Harrison’s Principles of Internal Medicine 20th ed. McGraw-Hill Education; 2018.

15. Bunschoten C, Jacobs BC, Van den Bergh PYK, et al. Progress in diagnosis and treatment of chronic inflammatory demyelinating polyradiculoneuropathy. Lancet Neurol 2019;18:784-794.

16. Van den Bergh PYK, Hadden RDM, Bouche P, et al. European Federation of Neurological Societies/Peripheral Nerve Society guideline on management of chronic inflammatory demyelinating polyradiculoneuropathy: report of a joint task force of the European Federation of Neurological Societies and the Peripheral Nerve Society-first revision. Eur J Neurol 2010;17:356-363.

17. Breiner A, Brannagan TH. Comparison of sensitivity and specificity among 15 criteria for chronic inflammatory demyelinating polyneuropathy. Muscle Nerve 2014;50:40-46.

18. Kuwabara S, Misawa S. Chronic inflammatory demyelinating polyneuropathy. Adv Exp Med Biol 2019;1190:333-343.

19. Benson AB, Venook AP, Al-Hawary MM, et al. NCCN guidelines insights: colon cancer, version 2.2018. J Natl Compr Canc Netw 2018; 16:359-369.

20. Kakushadze Z, Raghubanshi R, Yu W. Estimating cost savings from early cancer diagnosis. Data 2017;2:30. 